\title{
A GENERALIZATION OF PP-RINGS AND p.q.-BAER RINGS*
}

\author{
LIU ZHONGKUI and ZHAO RENYU \\ Department of Mathematics, Northwest Normal University, Lanzhou 730070, Gansu, \\ People's Republic of China \\ e-mail:liuzk@nwnu.edu.cn; renyuzhao026@sohu.com
}

(Received 6 June, 2005; revised 2 February, 2006; accepted 3 February, 2006)

\begin{abstract}
We introduce the concept of left APP-rings which is a generalization of left p.q.-Baer rings and right PP-rings, and investigate its properties. It is shown that the APP property is inherited by polynomial extensions and is a Morita invariant property.
\end{abstract}

2000 Mathematics Subject Classification. 16D40.

1. Introduction. Throughout this paper, $R$ denotes a ring with unity. Recall that $R$ is (quasi-) Baer if the right annihilator of every nonempty subset (every right ideal) of $R$ is generated by an idempotent of $R$. In [13] Kaplansky introduced Baer rings to abstract various properties of $A W^{*}$-algebras and von Neumann algebras. Clark defined quasi-Baer rings in [9] and used them to characterize when a finite dimensional algebra with unity over an algebraically closed field is isomorphic to a twisted matrix units semigroup algebra. As a generalization of quasi-Baer rings, Birkenmeier, Kim and Park in [6] introduced the concept of principally quasi-Baer rings. A ring $R$ is called left principally quasi-Baer (or simply left p.q.-Baer) if the left annihilator of a principal left ideal of $R$ is generated by an idempotent. Similarly, right p.q.-Baer rings can be defined. A ring is called p.q.-Baer if it is both right and left p.q.-Baer. Observe that biregular rings and quasi-Baer rings are p.q.-Baer. For more details and examples of left p.q.-Baer rings, see [3], [4], [5], [6], and [15]. We say a ring $R$ is a left APP-ring if the left annihilator $l_{R}(R a)$ is right s-unital as an ideal of $R$ for any element $a \in R$. This concept is a common generalization of left p.q.-Baer rings and right PP-rings. In this paper we investigate left APP-rings. In section 2 we provide several basic results. In section 3 we discuss various constructions and extensions under which the class of left APP-rings is closed.

For a nonempty subset $Y$ of $R, l_{R}(Y)$ and $r_{R}(Y)$ denote the left and right annihilator of $Y$ in $R$, respectively.

2. Left APP-rings. An ideal $I$ of $R$ is said to be right s-unital if, for each $a \in I$ there exists an element $x \in I$ such that $a x=a$. Note that if $I$ and $J$ are right $s$-unital ideals, then so is $I \cap J$ (if $a \in I \cap J$, then $a \in a I J \subseteq a(I \cap J)$ ). It follows from [22, Theorem 1] that $I$ is right s-unital if and only if for any finitely many elements $a_{1}, a_{2}, \ldots, a_{n} \in I$ there exists an element $x \in I$ such that $a_{i}=a_{i} x, i=1,2, \ldots, n$. A submodule $N$ of a left $R$-module $M$ is called a pure submodule if $L \otimes_{R} N \longrightarrow L \otimes_{R} M$ is a monomorphism

\footnotetext{
* Supported by National Natural Science Foundation of China (10171082), TRAPOYT and the Cultivation Fund of the Key Scientific and Technical Innovation Project, Ministry of Education of China.
} 
for every right $R$-module $L$. By [19, Proposition 11.3.13], an ideal $I$ is right s-unital if and only if $R / I$ is flat as a left $R$-module if and only if $I$ is pure as a left ideal of $R$.

Definition 2.1. A ring $R$ is called a left $A P P$-ring if the left annihilator $l_{R}(R a)$ is right s-unital as an ideal of $R$ for any element $a \in R$.

Right APP-rings may be defined analogously. Clearly every left p.q.-Baer ring is a left APP-ring (thus the class of left APP-rings includes all biregular rings and all quasi-Baer rings).

A ring $R$ is called a right (resp. left) PP-ring if the right (resp. left) annihilator of an element of $R$ is generated by an idempotent. $R$ is called a $P P$-ring if it is both right and left PP. Clearly every Baer ring is a PP-ring. The following result appeared in Fraser and Nicholson [10, Proposition 1].

LEMMA 2.2. The following conditions are equivalent for a ring $R$.

(1) $R$ is a right PP-ring.

(2) If $\emptyset \neq X \subseteq R$ then for all $a \in l_{R}(X), a \in a l_{R}(X)$.

From [1], a ring $R$ is called an Armendariz ring if whenever $f(x)=\sum_{i=0}^{m} a_{i} x^{i}, g(x)=$ $\sum_{j=0}^{n} b_{j} x^{j} \in R[x]$ satisfy $f(x) g(x)=0$, we have $a_{i} b_{j}=0$ for every $i$ and $j$. From [11], a ring $R$ is called a quasi-Armendariz ring if whenever $f(x)=\sum_{i=0}^{m} a_{i} x^{i}, g(x)=\sum_{j=0}^{n} b_{j} x^{j} \in$ $R[x]$ satisfy $f(x) R[x] g(x)=0$, we have $a_{i} R b_{j}=0$ for every $i$ and $j$. Armendariz rings are quasi-Armendariz rings. Results and examples of quasi-Armendariz rings appeared in [11].

PROPOSITION 2.3. For any ring, we have the following implications:

(1) right $P P \Rightarrow$ left $A P P$.

(2) quasi-Baer $\Rightarrow$ left p.q.-Baer $\Rightarrow$ left APP $\Rightarrow$ quasi-Armendariz.

Proof. (1). This follows from Lemma 2.2.

(2). If $R$ is a left APP-ring, then, by [11, Theorem 3.9], $R$ is a quasi-Armendariz ring. Other implications are clear.

All of the converses in Proposition 2.3 do not hold. In fact, left p.q.-Baer $\nRightarrow$ quasi-Baer follows from [6, Example 1.5]. Some examples were given in [6, Examples 1.3 and 1.5] to show that the class of left p.q.-Baer rings is not contained in the class of right PP-rings and, the class of right PP-rings is not contained in the class of left p.q.-Baer rings. By Proposition 2.3, it is clear that both of these classes are contained in the class of left APP-rings. This shows that left APP $\nRightarrow$ left p.q.-Baer and left APP $\nRightarrow$ right PP. Quasi-Armendariz $\nRightarrow$ left APP follows from the following example.

EXAMPLE 2.4. Use the ring in [4, Example 2.3]. For a given field $F$, let

$$
S=\left\{\left(a_{n}\right)_{n=1}^{\infty} \in \prod F \mid a_{n} \text { is eventually constant }\right\},
$$

which is a subring of the countably infinite direct product $\prod F$. Then the ring $S$ is a commutative ring. Let $R=S[[x]]$. Clearly $S$ is a reduced ring. Suppose that $f(x)=a_{0}+a_{1} x+a_{2} x^{2}+\cdots$ and $g(x)=b_{0}+b_{1} x+b_{2} x^{2}+\cdots \in S[[x]]$ are such that $f(x) g(x)=0$. Then, from [1, p. 2269], it follows that $a_{i} b_{j}=0$ for all $i$ and $j$. Thus $R$ is a reduced ring. From [2], $R$ is an Armendariz ring, and so it is a quasi-Armendariz ring. 
Suppose that $R$ is an APP-ring. Let $f(x)=f_{0}+f_{1} x+f_{2} x^{2}+\cdots$ and $g(x)=$ $g_{0}+g_{1} x+g_{2} x^{2}+\cdots \in R$, where $f_{0}=(0,1,0,0, \ldots), f_{1}=(0,1,0,1,0,0, \ldots), f_{2}=$ $(0,1,0,1,0,1,0,0, \ldots), \ldots$, and $g_{0}=(1,0,0,0, \ldots), g_{1}=(1,0,1,0,0,0, \ldots), g_{2}=$ $(1,0,1,0,1,0,0,0, \ldots), \ldots$ Then $g(x) \in l_{R}(R f(x))$. Thus there exists $h(x) \in l_{R}(R f(x))$ such that $g(x)=g(x) h(x)$. Suppose that $h(x)=h_{0}+h_{1} x+h_{2} x^{2}+\cdots$. Now from $h(x) f(x)=0$ and from [1, p. 2269] it follows that $h_{i} f_{j}=0$ for all $i$ and $j$ and, so there exists $n_{i} \in \mathbb{N}$ such that $h_{i}$ has the form $\left(b_{1}^{i}, 0, b_{3}^{i}, 0, \ldots, b_{2 n_{i}+1}^{i}, 0,0,0, \ldots\right)$, where $b_{k}^{i} \in F, i=0,1,2, \ldots$ From $g(x)(1-h(x))=0$ it follows that $g_{i}\left(1-h_{0}\right)=0$ and $g_{i} h_{j}=0$ for all $i$ and $j \geq 1$ and, so there exists $m_{i} \in \mathbb{N}$ such that $h_{i}$ has the form $\left(0, b_{2}^{i}, 0, b_{4}^{i}, 0, \ldots, b_{2 m_{i}}^{i}, 0,0,0, \ldots\right)$, where $b_{k}^{i} \in F, i=1,2, \ldots$ Thus $h_{1}=h_{2}=\cdots=$ 0 and so $h(x)=h_{0}$. This contradicts with $g_{i}=g_{i} h_{0}, i=0,1, \ldots$ Thus $R$ is not APP.

The following is an example of commutative APP-rings which are neither PP nor p.q.-Baer. Recall that a ring $R$ is called a left Bezout ring if every finitely generated left ideal of $R$ is principal. We denote by w.g. $\operatorname{dim}(R)$ the weak global dimension of a ring $R$, which is defined as $\sup \{f d(A) \mid A$ is a left $R$-module $\}$. Note that w.g.dim $(R) \leq 1$ if and only if every left ideal of $R$ is flat.

EXAMPLE 2.5. (see, [8, p. 64]) Let $\mathbb{Z}$ be the ring of integers and let

$$
S=\left(\prod_{i=1}^{\infty} \mathbb{Z} / 2 \mathbb{Z}\right) /\left(\bigoplus_{i=1}^{\infty} \mathbb{Z} / 2 \mathbb{Z}\right) .
$$

Then $S$ is clearly a Boolean ring and, by $[8$, p. 64], the weak global dimension of $S[[x]]$ is one and $S[[x]]$ is not semihereditary. Let $R=S[[x]]$. Then every principal ideal of $R$ is flat, and so $R / l_{R}(R a)=R / l_{R}(a) \cong R a$ is flat. Thus $l_{R}(R a)$ is pure as a left ideal of $R$ for every $a \in R$. Hence $R$ is an APP-ring. In [8, Theorem 43], it was shown that the power series ring $A[[x]]$ over a von Neumann regular ring $A$ is semihereditary if and only if $A[[x]]$ is a Bezout ring in which all principal ideals are projective. On the other hand, by [8, Theorem 42], $S[[x]]$ is a Bezout ring since the weak global dimension of $S[[x]]$ is one. Thus $R$ is not PP, and so is not p.q.-Baer.

Proposition 2.6. The following conditions are equivalent for a ring $R$.

(1) $R$ is a left APP-ring.

(2) If $I$ is a finitely generated left ideal of $R$ then for all $a \in l_{R}(I), a \in a l_{R}(I)$.

Proof. Clearly (2) implies (1). Now suppose that $R$ is a left APP-ring and $I=$ $R a_{1}+\cdots+R a_{n}$ is a finitely generated left ideal of $R$. Then $l_{R}(I)=\cap_{i=1}^{n} l_{R}\left(R a_{i}\right)$. Let $a \in l_{R}(I)$. Then $a \in l_{R}\left(R a_{i}\right)$ for each $i$. Hence there exists $x_{i} \in l_{R}\left(R a_{i}\right)$ such that $a x_{i}=a$ for each $i$. Then $a x=a$, where $x=x_{1} x_{2} \cdots x_{n} \in l_{R}(I)$.

Proposition 2.7. Suppose that $R$ satisfies the ascending chain condition on principal left ideals. Then the following conditions are equivalent.

(1) $R$ is a left APP-ring.

(2) $R$ is a left p.q.-Baer ring.

Proof. Clearly (2) implies (1). Suppose that $R$ is a left APP-ring. For every $a \in R$, denote $L=l_{R}(R a)$. Take a maximal principal ideal $R b$ contained in $L$. Since $b=b l$ for some $l \in L, R b \subseteq R l$, so maximality of $R b$ implies that $R b=R l$. Hence $l=x b$ for some $x \in R$ and $b=b x b$ and $R b=R e$, where $e=x b=e^{2}$. Clearly $L=L e+L(1-e)$. Note that if $t \in L(1-e)$, then $R e \subseteq R(e+t-e t) \subseteq L$. Hence $R e=R(e+t-e t)$ and, 
since $R e \cap R(t-e t)=0$, we get that $t-e t=0$. However $(e t)^{2}=0$, so $t^{2}=0$. On the other hand, for every $u \in L(1-e), u=u l$ for some $l \in L$. Consequently $u=u l(1-e)$. Now $w=l(1-e) \in L(1-e)$, so $w^{2}=0$. Consequently $u=u w=u w^{2}=0$. Thus $L(1-e)=0$, so $L=R e$ and we are done.

Note that this reasoning shows in fact that in rings satisfying ascending chain condition on principal left ideals, right $s$-unital ideals are generated by idempotents (as left ideals).

Proposition 2.8. Let R be a commutative Bezout ring. Then the following conditions are equivalent.

(1) $R$ is an APP-ring.

(2) w.g. $\operatorname{dim}(R) \leq 1$.

Proof. If $R$ is a commutative Bezout ring, then w.g.dim $(R) \leq 1$ if and only if every ideal of $R$ is flat if and only if every finitely generated ideal of $R$ is flat if and only if every principal ideal of $R$ is flat if and only if $R / l_{R}(R a)$ is flat for every $a \in R$ if and only if $R$ is an APP-ring.

Note that Baer rings have no nonzero central nilpotent elements, and so commutative Baer rings are reduced. Huh, Kim and Lee in [12, Proposition 4] extended this property onto right PP-rings by showing that right PP-rings have no nonzero central nilpotent elements. For left APP-rings we have the following more general result.

Proposition 2.9. Let $R$ be a left APP-ring. If $0 \neq a \in R$ is such that $l_{R}(R a) \subseteq r_{R}(a)$, then $a R a \neq 0$.

Proof. Suppose that $a R a=0$. Then $a \in l_{R}(R a)$. Since $R$ is a left APP-ring, there exists $b \in l_{R}(R a)$ such that $a=a b$. Thus $b \in r_{R}(a)$ and so $a=a b=0$.

As a corollary we have that left APP-rings have no nonzero central nilpotent elements.

COROLlary 2.10. Let $R$ be a left APP-ring. Then $R$ is semiprime if and only if $l_{R}(R a) \subseteq r_{R}(a)$ for all $a \in R$.

Proof. Suppose that $R$ is semiprime. Note that $\left((R a) l_{R}(R a) R\right)^{2}=0$ for all $a \in R$. Thus $\operatorname{Ral}_{R}(R a) R=0$ and so $l_{R}(R a) \subseteq r_{R}(a)$ for all $a \in R$. Conversely if $l_{R}(R a) \subseteq r_{R}(a)$ for all $a \in R$, then, by Proposition 2.9, $R$ is semiprime.

Corollary 2.11. Commutative APP-rings are reduced.

In [12, Example 3], an example was given to show that commutative reduced rings need not be PP. In fact, there exist commutative reduced rings which need not be APP. For example, let $R$ be the ring as in Example 2.4. Then $R$ is a commutative reduced ring. But $R$ is not an APP-ring.

3. Extensions of left APP-rings. In this section we discuss various constructions and extensions under which the class of left APP-rings is closed. We deal with the direct sums as rings without identity when the index sets are infinite. In this case the definitions of right PP-rings, left p.q.-Baer rings and left APP-rings are also valid. 
Note that the direct sums of right PP-rings need not be right PP. Consider the following example. Let $F$ be a field and $R_{i}=F, i=1,2, \ldots$ Suppose that $R=\oplus_{i=1}^{\infty} R_{i}$ is a right PP-ring. Then for $a=(1,0,0, \cdots) \in R$, there exists $e \in R$ such that $r_{R}(a)=$ $e R$. Write $e=\left(e_{1}, e_{2}, \ldots, e_{n}, 0,0, \ldots\right)$. Denote $x=\left(x_{i}\right)_{i=1}^{\infty}$ where $x_{n+1}=1$ and $x_{i}=0$ for $i=1,2, \ldots, n, n+2, \ldots$ Clearly $a x=0$ but $x \notin e R$. So $R$ is not a PP-ring. This example also shows that the direct sums of left p.q.-Baer rings need not be left p.q.-Baer.

From [12], a ring $R$ is called a generalized right PP-ring if for any $x \in R$ the right ideal $x^{n} R$ is projective for some positive integer $n$, depending on $x$, or equivalently, if for any $x \in R$ the right annihilator of $x^{n}$ is generated by an idempotent for some positive integer $n$, depending on $x$. By [12, Lemma 1(iv)], $R$ is a generalized right PP-ring if and only if $R$ is a right PP-ring when $R$ is reduced. Note that in the above example, the ring $R=\oplus_{i=1}^{\infty} R_{i}$ is reduced. So above example also shows that the direct sums of generalized right PP-rings need not be generalized right PP. Hence Proposition 7(ii) of [12] is incorrect.

But for left APP-rings we have the following result.

Proposition 3.1. Let $R_{i}, i \in I$ be rings. Then we have the following:

(1) $R=\prod_{i \in I} R_{i}$ is a left APP-ring if and only if $R_{i}$ is a left APP-ring for each $i \in I$.

(2) $R=\bigoplus_{i \in I} R_{i}$ is a left APP-ring if and only if $R_{i}$ is a left APP-ring for each $i \in I$.

If $|I|<\infty$, then the result is clear. If $|I|=\infty$, then Proposition 3.1 is a direct corollary of the following more general result. Let $\aleph$ be an infinite cardinal number. Suppose that $I$ is a set and $\left\{R_{i} \mid i \in I\right\}$ is a family of rings. Let $x=\left(x_{i}\right)_{i \in I} \in \prod_{i \in I} R_{i}$. We define the support of $x$ as $\operatorname{supp}(x)=\left\{i \in I \mid x_{i} \neq 0\right\}$. For an infinite cardinal number $\aleph$, define the $\aleph$-product of the $R_{i}$ 's as

$$
\prod_{i \in I}^{\aleph} R_{i}=\left\{x \in \prod_{i \in I} R_{i}|| \operatorname{supp}(x) \mid<\aleph\right\} .
$$

Clearly one may view the direct sum and the direct product of a family of rings as two special cases of the same object, namely, the $\aleph$-product of the family of rings. $\aleph$-products of some families of modules have been studied by [17], [20] and [21].

Proposition 3.2. Let $R_{i}, i \in I$ be rings. Then $R=\prod_{i \in I}^{\aleph} R_{i}$ is a left APP-ring if and only if $R_{i}$ is a left APP-ring for each $i \in I$.

Proof. If the ring $R$ is a left APP-ring, then clearly so is each $R_{i}$. Conversely suppose that every $R_{i}$ is a left APP-ring. Let $a=\left(a_{i}\right)_{i \in I}$ and $b=\left(b_{i}\right)_{i \in I}$ be in $R$ such that $a R b=0$. Then $a_{i} R_{i} b_{i}=0$ for every $i \in I$. Thus, for every $i \in \operatorname{supp}(b)$, there exists $c_{i} \in R_{i}$ such that $a_{i}=a_{i} c_{i}$ and $c_{i} R_{i} b_{i}=0$. Now define $x=\left(x_{i}\right)_{i \in I}$ via

$$
x_{i}=\left\{\begin{array}{l}
c_{i} i \in \operatorname{supp}(b) \\
1 \quad i \in \operatorname{supp}(a)-\operatorname{supp}(b) \\
0 \quad i \notin \operatorname{supp}(a) \cup \operatorname{supp}(b) .
\end{array}\right.
$$

Then $x \in R$ since $|\operatorname{supp}(x)|<\aleph$, and $a=a x, x R b=0$. Thus $R$ is a left APP-ring.

Let $A$ be a ring, $B$ be a unitary subring of $A,\left\{A_{i}\right\}_{i=1}^{\infty}$ be a countable set of copies of $A, D$ be the direct product of all rings $A_{i}$, and let $R=R(A, B)$ be the subring of $D$ generated by the ideal $\bigoplus_{i=1}^{\infty} A_{i}$ and by the subring $\{(b, b, \cdots) \mid b \in B\}$ (see [23]). Then we have the following result. 
PROPOSITION 3.3. If $A$ is a commutative ring, then the ring $R(A, B)$ is an $A P P$-ring if and only if $A$ and $B$ are APP-rings.

Proof. Denote $R=R(A, B)$. Let $\left(x_{i}\right)_{i=1}^{\infty}$ and $\left(y_{i}\right)_{i=1}^{\infty} \in R$ be such that $\left(x_{i}\right)_{i=1}^{\infty} R\left(y_{i}\right)_{i=1}^{\infty}=0$. We note that there exists $n$ such that $x_{n}=x_{n+1}=\cdots \in B$ and $y_{n}=y_{n+1}=\cdots \in B$. Clearly we have $x_{i} A y_{i}=0$ for $i=1,2, \ldots, n$. Since $A$ is an APP-ring, there exists $w_{i} \in A$ such that $x_{i}=x_{i} w_{i}$ and $w_{i} A y_{i}=0, i=1,2, \ldots, n-1$. Since $B$ is an APP-ring and $x_{n} B y_{n}=0$, there exists $w_{n} \in B$ such that $x_{n}=x_{n} w_{n}$ and $w_{n} B y_{n}=0$. Since $A$ is commutative, we have $w_{n} A y_{n}=0$. Thus $\left(x_{i}\right)_{i=1}^{\infty}=$ $\left(x_{i}\right)_{i=1}^{\infty}\left(w_{1}, w_{2}, \ldots, w_{n-1}, w_{n}, w_{n}, \ldots\right)$ and $\left(w_{1}, w_{2}, \ldots, w_{n-1}, w_{n}, w_{n}, \ldots\right) R\left(y_{i}\right)_{i=1}^{\infty}=0$. Hence $R$ is an APP-ring.

Conversely, if $R$ is an APP-ring, then it is easy to see that $A$ and $B$ are APP-rings by noting that $A$ is commutative.

Note that if $R(A, B)$ is a left APP-ring, then $A$ is a left APP-ring. But Example 3.9(2) shows that $B$ need not be a left APP-ring in general.

Proposition 3.4. Let $A$ be a left $A P P$-ring. If $l_{B}(A b)=0$ for every $0 \neq b \in B$, then the ring $R(A, B)$ is a left $A P P$-ring.

Proof. In the proof of Proposition 3.3, if $y_{n}=0$, then take $w_{n}=1 \in B$. If $y_{n} \neq 0$, then $l_{B}\left(A y_{n}\right)=0$. Thus $x_{n}=0$. If we take $w_{n}=0$, then $x_{n}=x_{n} w_{n}$ and $w_{n} A y_{n}=0$. Thus $\left(x_{i}\right)_{i=1}^{\infty}=\left(x_{i}\right)_{i=1}^{\infty}\left(w_{1}, w_{2}, \ldots, w_{n-1}, w_{n}, w_{n}, \ldots\right)$ and $\left(w_{1}, w_{2}, \ldots, w_{n-1}\right.$, $\left.w_{n}, w_{n}, \ldots\right) R\left(y_{i}\right)_{i=1}^{\infty}=0$. Hence $R$ is a left APP-ring.

Let $n$ be a positive integer. Let $M_{n}(R)$ denote the ring of $n \times n$ matrices over $R$.

Proposition 3.5. $R$ is a left APP-ring if and only if $M_{n}(R)$ is a left APP-ring.

Proof. Let $R$ be a left APP-ring and $A=\left(a_{i j}\right) \in M_{n}(R)$. Suppose that $B=\left(b_{i j}\right) \in$ $M_{n}(R)$ is such that $B \in l_{M_{n}(R)}\left(M_{n}(R) A\right)$. Then $B M_{n}(R) A=0$. Let $E_{i j}$ denote the $(i, j)$ matrix unit. Then $\left(\sum_{p, q} b_{p q} E_{p q}\right) r E_{i j}\left(\sum_{s, t} a_{s t} E_{s t}\right)=0$ for any $r \in R$ and any $i$ and $j$. Thus $\sum_{p, t} b_{p i} r a_{j t} E_{p t}=0$, which implies that $b_{p i} r a_{j t}=0$ for any $p$ and $t$. Hence $b_{p i} \in l_{R}\left(R a_{j t}\right)$ for all $i, j, p$ and $t$. So $b_{p q} \in l_{R}\left(\sum_{i, j} R a_{i j}\right)$ for all $p, q$. By Proposition 2.6, there exists $c \in l_{R}\left(\sum_{i, j} R a_{i j}\right)$ such that $b_{p q}=b_{p q} c$ for all $p, q$. Thus

$$
B=B\left(\begin{array}{lll}
c & & \\
& c & \\
& \ddots & \\
& & c
\end{array}\right)
$$

and it is easy to see that

$$
\left(\begin{array}{llll}
c & & \\
& c & & \\
& & \ddots & \\
& & & c
\end{array}\right) M_{n}(R) A=0 .
$$

Thus $M_{n}(R)$ is a left APP-ring. 
Conversely suppose that $M_{n}(R)$ is a left APP-ring and $a, b \in R$ is such that $a \in$ $l_{R}(R b)$. Set

$$
A=\left(\begin{array}{llll}
a & & & \\
& 0 & & \\
& \ddots & \\
& & & 0
\end{array}\right), \quad B=\left(\begin{array}{llll}
b & & & \\
& 0 & & \\
& & \ddots & \\
& & & 0
\end{array}\right) .
$$

Then $A M_{n}(R) B=0$. Thus there exists $C=\left(c_{i j}\right) \in M_{n}(R)$ such that $A=A C$, $C M_{n}(R) B=0$. Now it is easy to see that $a=a c_{11}$ and $c_{11} R b=0$. Thus $R$ is a left APP-ring.

Proposition 3.6. $R$ is a left APP-ring if and only if the upper triangular matrix ring $T_{n}(R)$ over $R$ is a left APP-ring.

Proof. Let $R$ be a left APP-ring and $A=\left(a_{i j}\right) \in T_{n}(R)$. Suppose that $B=\left(b_{i j}\right) \in$ $T_{n}(R)$ is such that $B \in l_{T_{n}(R)}\left(T_{n}(R) A\right)$. Then $B T_{n}(R) A=0$. By analogy with the proof of Proposition 3.5, we obtain that $b_{p i} \in l_{R}\left(R a_{j t}\right)$ for all $i, j, p$ and $t$ with $p \leq i \leq j \leq t$. Thus $b_{11} \in l_{R}\left(\sum_{1 \leq i \leq j \leq n} R a_{i j}\right), b_{12}, b_{22} \in l_{R}\left(\sum_{2 \leq i \leq j \leq n} R a_{i j}\right), \ldots, b_{1, n-1}, b_{2, n-1}, \ldots, b_{n-1, n-1} \in$ $l_{R}\left(\sum_{n-1 \leq i \leq j \leq n} R a_{i j}\right), b_{1 n}, b_{2 n}, \ldots, b_{n n} \in l_{R}\left(R a_{n n}\right)$. Since $R$ is a left APP-ring, by Proposition 2.6, there exist $c_{1}, c_{2}, \ldots, c_{n}$ such that

$$
\begin{aligned}
& c_{1} \in l_{R}\left(\sum_{1 \leq i \leq j \leq n} R a_{i j}\right), \quad b_{11}=b_{11} c_{1}, \\
& c_{2} \in l_{R}\left(\sum_{2 \leq i \leq j \leq n} R a_{i j}\right), \quad b_{12}=b_{12} c_{2}, b_{22}=b_{22} c_{2}, \\
& \ldots \ldots \ldots \ldots \\
& c_{n} \in l_{R}\left(R a_{n n}\right), \quad b_{k n}=b_{k n} c_{n}, k=1,2, \ldots, n .
\end{aligned}
$$

Now it is easy to see that

$$
B=B\left(\begin{array}{llll}
c_{1} & & & \\
& c_{2} & & \\
& & \ddots & \\
& & & c_{n}
\end{array}\right),\left(\begin{array}{llll}
c_{1} & & & \\
& c_{2} & & \\
& & \ddots & \\
& & & c_{n}
\end{array}\right) T_{n}(R) A=0 .
$$

Hence $T_{n}(R)$ is a left APP-ring.

Conversely if $T_{n}(R)$ is a left APP-ring, then, by analogy with the proof of Proposition 3.5, we can show that $R$ is left APP.

Proposition 3.7. Let $e \in R$ be an idempotent. If $R$ is a left APP-ring then eRe is a left APP-ring.

Proof. Let $x \in e$ Re and $a \in l_{e R e}(e \operatorname{Rex})$. Then aRexe $=(a e) \operatorname{Rexe}=a(e \operatorname{Re}) x e=0$. Thus $a \in l_{R}(R($ exe $))$. Since $l_{R}(R($ exe $))$ is pure as a left ideal of $R$, there exists $b \in l_{R}(R(e x e))$ such that $a=a b$. Thus $a=a e=a b e=(e a e) b e=(e a e)(e b e)=a(e b e)$ and $(e b e)(e R e) x=e b(e R e) x=e b(e R e) x e=e b(e R)(e x e) \subseteq e b R(e x e)=0$. Hence $e b e \in$ $l_{e R e}(e \operatorname{Rex})$. This means that $l_{e R e}(e \operatorname{Rex})$ is pure as a left ideal of $e \operatorname{Re}$ and so $e$ Re is a left APP-ring. 
From [6, Theorem 2.2], the concept of left p.q.-Baer rings is a Morita invariant property. But the concept of right PP-rings is not a Morita invariant property because $\mathbb{Z}[x]$ is Baer but the $2 \times 2$ full matrix ring over $\mathbb{Z}[x]$ is not a right PP-ring ([2]). From Propositions 3.5 and 3.7, for left APP-rings we have the following result.

THEOREM 3.8. The endomorphism ring of a finitely generated projective module over a left APP-ring is left APP. In particular, the left APP condition is a Morita invariant property.

EXAMPLE 3.9. (1). Subrings of a left APP-ring need not be left APP. Let $A=M_{2}(F)$ where $F$ is a field. Then $A$ is a left APP-ring by Proposition 3.5. Let

$$
B=\left\{\left(\begin{array}{ll}
a & b \\
0 & a
\end{array}\right) \mid a, b \in F\right\} .
$$

Then $B$ is not a left APP-ring by Proposition 2.9.

(2). Factor rings of a left APP-ring need not be left APP. The ring $\mathbb{Z}$ of integers is an APP-ring whereas its homomorphic image $\mathbb{Z} / 4 \mathbb{Z}$ is not. The following is another example of such rings. Let $A, B$ be as in (1). Suppose that $a, b, c, d \in F$ are such that

$$
\left(\begin{array}{ll}
a & b \\
0 & a
\end{array}\right) A\left(\begin{array}{ll}
c & d \\
0 & c
\end{array}\right)=0
$$

but $\left(\begin{array}{ll}c & d \\ 0 & c\end{array}\right) \neq 0$. If $c \neq 0$, then clearly $\left(\begin{array}{ll}a & b \\ 0 & a\end{array}\right)=0$. If $c=0$, then $d \neq 0$. From

$$
\left(\begin{array}{ll}
a & b \\
0 & a
\end{array}\right)\left(\begin{array}{ll}
0 & 0 \\
1 & 1
\end{array}\right)\left(\begin{array}{ll}
0 & d \\
0 & 0
\end{array}\right)=0
$$

it follows that $b d=0$ and $a d=0$, which imply that $a=b=0$. Thus, $\left.l_{B}\left(\begin{array}{ll}A & d \\ 0 & c\end{array}\right)\right)=0$, and so, by Proposition 3.4, $R=R(A, B)$ is a left APP-ring since $A$ is a left APP-ring. But the factor ring $R /\left(\oplus_{i=1}^{\infty} A_{i}\right)$, which is isomorphic to $B$ by [23, Example 15.7(2)], is not left APP. This example also shows that if $R(A, B)$ is a left APP-ring, then $B$ need not be a left APP-ring in general.

Note that the ring $R=\left\{\left(\begin{array}{ll}a & b \\ 0 & a\end{array}\right) \mid a, b \in F\right\}$, where $F$ is a given field, is a generalized right PP-ring by [12, Proposition 3]. So Example 3.9(1) shows that generalized right PP-rings need not be left APP. On the other hand, let $R=M_{2}(\mathbb{Z}[x])$. Then $R$ is both left and right APP by Proposition 3.5 and Corollary 3.12. But $R$ is not a generalized right PP-ring by [12, Example 4]. Thus left APP-rings need not be generalized right PP.

Recall that a monoid $S$ is called a u.p.-monoid (unique product monoid) if for any two nonempty finite subsets $A, B \subseteq S$ there exists an element $g \in S$ uniquely presented in the form $a b$ where $a \in A$ and $b \in B$. The class of u.p.-monoids is quite large and important (see [7], [16] and [18]). For example, this class includes the right or left ordered monoids, submonoids of a free group, and torsion-free nilpotent groups. Every u.p.-monoid $S$ has no non-unity element of finite order.

Let $R$ be a ring and $S$ a u.p.-momoid. Assume that there is a monoid homomorphism $\alpha: S \longrightarrow \operatorname{Aut}(R)$. For any $s \in S$, we denote the image of $s$ under $\alpha$ by $\alpha_{s}$. Then we can form a skew monoid ring $R * S$ (induced by the monoid homomorphism $\alpha$ ) by taking its elements to be finite formal combinations $\sum_{s \in S} a_{s} s$, with multiplication 
induced by:

$$
\left(a_{s} s\right)\left(b_{t} t\right)=a_{s} \alpha_{s}\left(b_{t}\right)(s t) .
$$

A monoid homomorphism $\alpha: S \longrightarrow \operatorname{Aut}(R)$ is said to satisfy condition (*) if for every $a \in R$, the left ideal $\sum_{s \in S} R \alpha_{s}(a)$ is finitely generated. In [11, Theorem 3.9], it was shown that a ring $R$ is left APP if and only if $R[x]$ is left APP. For skew monoid rings we have the following result.

THEOREM 3.10. Let $R$ be a left APP-ring and $S$ a u.p.-monoid. If $\alpha: S \longrightarrow \operatorname{Aut}(R)$ satisfies the condition $(*)$, then the skew monoid ring $R * S$ (induced by the monoid homomorphism $\alpha$ ) is a left APP-ring.

Proof. Suppose that $f=a_{1} s_{1}+a_{2} s_{2}+\cdots+a_{n} s_{n}, g=b_{1} t_{1}+b_{2} t_{2}+\cdots+b_{m} t_{m} \in$ $R * S$ are such that $f \in l_{R * S}((R * S) g)$. Then $f(R * S) g=0$. Thus for every $s \in S$ and every $r \in R, f(r s) g=0$. Suppose that $c_{1}, c_{2}, \ldots, c_{n} \in R$ are such that $a_{i}=\alpha_{s_{i}}\left(c_{i}\right)$ for $i=1,2, \ldots, n$. We will show that $c_{i} \in l_{R}\left(R \alpha_{s}\left(b_{j}\right)\right)$ for every $s \in S, i=1,2, \ldots, n, j=$ $1,2, \ldots, m$ by induction on $m$.

If $m=1$, then $g=b_{1} t_{1}$. Thus $0=\left(a_{1} s_{1}+a_{2} s_{2}+\cdots+a_{n} s_{n}\right)(r s)\left(b_{1} t_{1}\right)=a_{1} \alpha_{s_{1}}$ $\left(r \alpha_{s}\left(b_{1}\right)\right) s_{1} s t_{1}+a_{2} \alpha_{s_{2}}\left(r \alpha_{s}\left(b_{1}\right)\right) s_{2} s t_{1}+\cdots+a_{n} \alpha_{s_{n}}\left(r \alpha_{s}\left(b_{1}\right)\right) s_{n} s t_{1}$ for every $r \in R$. By [7, Lemma 1.1], $S$ is a cancellative monoid. Thus $s_{i} s t_{1} \neq s_{j} s t_{1}$ for $s_{i} \neq s_{j}$. Hence $a_{i} \alpha_{s_{i}}\left(r \alpha_{s}\left(b_{1}\right)\right)=0$, which implies that $c_{i} \in l_{R}\left(R \alpha_{s}\left(b_{1}\right)\right)$ since $\alpha_{s_{i}}$ is an automorphism, $i=1,2, \ldots, n$.

Now suppose that $m \geq 2$. Since $S$ is a u.p.-monoid, there exist $p, q$ with $1 \leq p \leq n$ and $1 \leq q \leq m$ such that $s_{p} s t_{q}$ is uniquely presented by considering two subsets $\left\{s_{1}, s_{2}, \ldots, s_{n}\right\}$ and $\left\{s t_{1}, s t_{2}, \ldots, s t_{m}\right\}$ of $S$. Thus from $f(r s) g=0$ it follows that $a_{p} \alpha_{s_{p}}\left(r \alpha_{s}\left(b_{q}\right)\right) s_{p} s t_{q}=0$ and so $a_{p} \alpha_{s_{p}}\left(r \alpha_{s}\left(b_{q}\right)\right)=0$. Thus $\alpha_{s_{p}}\left(c_{p} r \alpha_{s}\left(b_{q}\right)\right)=0$, which implies that $c_{p} r \alpha_{s}\left(b_{q}\right)=0$ for every $r \in R$ since $\alpha_{s_{p}}$ is an automorphism. Hence $c_{p} \in l_{R}\left(R \alpha_{s}\left(b_{q}\right)\right)$. Since $l_{R}\left(R \alpha_{s}\left(b_{q}\right)\right)$ is pure as a left ideal of $R$, there exists an element $e_{q} \in l_{R}\left(R \alpha_{s}\left(b_{q}\right)\right)$ such that $c_{p}=c_{p} e_{q}$. Thus for every $r \in R$, we have

$$
\begin{aligned}
0= & f\left(e_{q} r s\right) g=\left(a_{1} s_{1}+a_{2} s_{2}+\cdots+a_{n} s_{n}\right)\left(e_{q} r s\right) \\
& \cdot\left(b_{1} t_{1}+b_{2} t_{2}+\cdots+b_{q-1} t_{q-1}+b_{q+1} t_{q+1}+\cdots+b_{m} t_{m}\right) \\
& +\left(a_{1} s_{1}+a_{2} s_{2}+\cdots+a_{n} s_{n}\right)\left(\left(e_{q} r \alpha_{s}\left(b_{q}\right)\right) s t_{q}\right) \\
= & \left(a_{1} \alpha_{s_{1}}\left(e_{q}\right) s_{1}+a_{2} \alpha_{s_{2}}\left(e_{q}\right) s_{2}+\cdots+a_{n} \alpha_{s_{n}}\left(e_{q}\right) s_{n}\right)(r s) \\
& \cdot\left(b_{1} t_{1}+b_{2} t_{2}+\cdots+b_{q-1} t_{q-1}+b_{q+1} t_{q+1}+\cdots+b_{m} t_{m}\right) .
\end{aligned}
$$

Since $a_{i} \alpha_{s_{i}}\left(e_{q}\right)=\alpha_{s_{i}}\left(c_{i} e_{q}\right)$, by induction, it follows that $c_{i} e_{q} \in l_{R}\left(R \alpha_{s}\left(b_{j}\right)\right)$ for $i=$ $1,2, \ldots, n, j=1,2, \ldots, q-1, q+1, \ldots, m$. Therefore

$$
c_{p}=c_{p} e_{q} \in \cap_{j=1}^{m} l_{R}\left(R \alpha_{s}\left(b_{j}\right)\right) .
$$

Now $a_{p} \alpha_{s_{p}}\left(R \alpha_{s}\left(b_{j}\right)\right)=\alpha_{s_{p}}\left(c_{p} R \alpha_{s}\left(b_{j}\right)\right)=0$ for any $j=1,2, \ldots, m$. Thus from $f(r s) g=0$ it follows that

$$
\begin{aligned}
0= & \left(a_{1} s_{1}+a_{2} s_{2}+\cdots+a_{p-1} s_{p-1}+a_{p+1} s_{p+1}+\cdots+a_{n} s_{n}\right) \\
& \cdot(r s)\left(b_{1} t_{1}+b_{2} t_{2}+\cdots+b_{m} t_{m}\right) .
\end{aligned}
$$

By using the previous method, there exists $k \in\{1,2, \ldots, p-1, p+1, \ldots, n\}$ such that $c_{k} \in \cap_{j=1}^{m} l_{R}\left(R \alpha_{s}\left(b_{j}\right)\right)$. Thus $a_{k} \alpha_{s_{k}}\left(R \alpha_{s}\left(b_{j}\right)\right)=\alpha_{s_{k}}\left(c_{k} R \alpha_{s}\left(b_{j}\right)\right)=0$ for any $j=1,2, \ldots, m$. 
Hence $\left(a_{1} s_{1}+a_{2} s_{2}+\cdots+a_{p-1} s_{p-1}+a_{p+1} s_{p+1}+\cdots+a_{k-1} s_{k-1}+a_{k+1} s_{k+1}+\cdots+a_{n} s_{n}\right)$ $(r s)\left(b_{1} t_{1}+b_{2} t_{2}+\cdots+b_{m} t_{m}\right)=0$. Continuing this procedure yields $c_{1}, c_{2}, \ldots, c_{n} \in$ $\bigcap_{j=1}^{m} l_{R}\left(R \alpha_{s}\left(b_{j}\right)\right)$ for every $s \in S$.

Set

$$
L=\sum_{j=1}^{m} \sum_{s \in S} R \alpha_{s}\left(b_{j}\right) .
$$

Then $c_{1}, c_{2}, \ldots, c_{n} \in l_{R}(L)$. Since $\alpha$ satisfies the condition $(*)$, it is easy to see that $L$ is finitely generated. From Proposition 2.6, $l_{R}(L)$ is pure as a left ideal of $R$. Thus there exists $d \in l_{R}(L)$ such that $c_{i}=c_{i} d, i=1,2, \ldots, n$. Denote by $\eta$ the identity of the monoid $S$. Then $f(d \eta)=\sum_{i=1}^{n} a_{i} \alpha_{s_{i}}(d) s_{i}=\sum_{i=1}^{n} \alpha_{s_{i}}\left(c_{i} d\right) s_{i}=$ $\sum_{i=1}^{n} \alpha_{s_{i}}\left(c_{i}\right) s_{i}=\sum_{i=1}^{n} a_{i} s_{i}=f$. For every $r \in R$ and every $s \in S, r \alpha_{s}\left(b_{j}\right) \in L$ and, so $(d \eta)(r s) g=\sum_{j=1}^{m} d r \alpha_{s}\left(b_{j}\right)\left(s t_{j}\right)=0$. Thus $d \eta \in l_{R * S}((R * S) g)$. This shows that $R * S$ is a left APP-ring.

REMARK 3.11. It is natural to ask for examples of monoid homomorphisms $\alpha$ : $S \longrightarrow \operatorname{Aut}(R)$ which satisfy the condition $\left(^{*}\right)$.

1. If $\alpha(s)=1$ for every $s \in S$, then $\alpha$ satisfies the condition $(*)$.

2. Let $T$ be a ring and $R=T \oplus T$. Let $\gamma: R \longrightarrow R$ be an automorphism defined by $\gamma((a, b))=(b, a)$. Let $S=\mathbb{Z}$ (or $S=\mathbb{N} \cup\{0\}$ ). Define $\alpha: S \longrightarrow \operatorname{Aut}(R)$ via $\alpha_{0}=1$ and $\alpha_{n}=\gamma^{n}$ for every $0 \neq n \in \mathbb{Z}$. Then $\sum_{n \in \mathbb{Z}} R \alpha_{n}((a, b))=R(a, b)+R(b, a)$ for every $(a, b) \in R$. Thus $\alpha$ is a monoid homomorphism satisfying the condition $(*)$.

3. Let $T$ be a ring and $R=M_{2}(T)$. Let $\gamma: R \longrightarrow R$ be an automorphism defined by

$$
\gamma\left(\left(\begin{array}{ll}
a & b \\
c & d
\end{array}\right)\right)=\left(\begin{array}{rr}
a & -b \\
-c & d
\end{array}\right)
$$

Let $S=\mathbb{Z}$ (or $S=\mathbb{N} \cup\{0\}$ ). Define $\alpha: S \longrightarrow \operatorname{Aut}(R)$ via $\alpha_{0}=1$ and $\alpha_{n}=\gamma^{n}$ for every $0 \neq n \in \mathbb{Z}$. Then

$$
\sum_{n \in \mathbb{Z}} R \alpha_{n}\left(\left(\begin{array}{ll}
a & b \\
c & d
\end{array}\right)\right)=R\left(\begin{array}{ll}
a & b \\
c & d
\end{array}\right)+R\left(\begin{array}{rr}
a & -b \\
-c & d
\end{array}\right)
$$

Thus $\alpha$ satisfies the condition $(*)$.

4. Let

$$
R=\left\{\left(\begin{array}{ll}
a & q \\
0 & a
\end{array}\right) \mid a \in \mathbb{Z}, q \in \mathbb{Q}\right\}
$$

Define $\gamma: R \longrightarrow R$ via

$$
\gamma\left(\left(\begin{array}{ll}
a & q \\
0 & a
\end{array}\right)\right)=\left(\begin{array}{ll}
a & 2 q \\
0 & a
\end{array}\right)
$$

Then it is easy to see that $\sum_{n=0}^{\infty} R \gamma^{n}\left(\left(\begin{array}{ll}a & q \\ 0 & a\end{array}\right)\right)=R\left(\begin{array}{ll}a & q \\ 0 & a\end{array}\right)$. Thus $\alpha: S=\mathbb{N} \cup\{0\} \longrightarrow$ $\operatorname{Aut}(R)$, defined by $\alpha_{0}=1$ and $\alpha_{n}=\gamma^{n}$ for every $n \in \mathbb{N}$, satisfies the condition (*).

Armendariz showed that polynomial rings over right PP-rings need not be right $\mathrm{PP}$ in the example in [2]. From [5, Theorem 2.1], a ring $R$ is a left p.q.-Baer ring if and only if $R[x]$ is a left p.q.-Baer ring. It was shown in [7, Corollary 1.4] that $R$ is left 
p.q.-Baer if and only if $R\left[x, x^{-1}\right]$ is left p.q.-Baer. For monoid rings, it was shown that the monoid ring $R[S]$ of a u.p.-monoid $S$ over a ring $R$ is left p.q.-Baer if and only if $R$ is left p.q.-Baer (see [7, Theorem 1.2]) and $R[S]$ is a reduced PP-ring if and only if $R$ is a reduced PP-ring (see [7, Corollary 1.3]). For left APP-rings we have the following result.

COROLlary 3.12. Let $S$ be a u.p.-monoid and $X$ a nonempty set of not necessarily commuting indeterminates. Then the following conditions are equivalent.

(1) $R$ is left $A P P$.

(2) $R[X]$ is left $A P P$.

(3) $R\left[x, x^{-1}\right]$ is left APP.

(4) $R[S]$ is left $A P P$.

Proof. The implication (1) $\Rightarrow$ (4) follows from Theorem 3.10. (4) $\Rightarrow$ (1). Let $a, b \in R$ be such that $a \in l_{R}(R b)$. Then $a \in l_{R[S]}(R[S] b)$. Thus there exists $\sum_{i=0}^{n} a_{i} s_{i} \in l_{R[S]}(R[S] b)$ with $s_{0}=\eta$, the identity of $S$, such that $a=a\left(\sum_{i=0}^{n} a_{i} s_{i}\right)$. Now it is easy to see that $a=a a_{0}$ and $a_{0} R b=0$. Thus $R$ is a left APP-ring.

$(1) \Leftrightarrow(2) \Leftrightarrow$ (3) follow from (1) $\Leftrightarrow(4)$, noting that the monoid generated by $X$ is a u.p.-monoid and $R\left[x, x^{-1}\right] \cong R[\mathbb{Z}]$, the monoid ring of the u.p.-monoid $\mathbb{Z}$ over $R$.

Corollary 3.13. Let $R$ be a left APP-ring and $\alpha$ a ring automorphism of $R$ such that $\sum_{i=0}^{\infty} R \alpha^{i}(b)$ is finitely generated for every $b \in R$. Then the skew polynomial ring $R[x ; \alpha]$ is a left APP-ring.

There exists a commutative von Neumann regular ring $R$ (hence left APP), but the ring $R[[x]]$ is not APP. For example, let $R$ be the ring $S$ defined in Example 2.4. Then $R$ is a commutative von Neumann regular ring. By Example 2.4, $R[[x]]$ is not an APP-ring.

Some additional conditions were given in [10], [14] and [15] for right PP-rings (or left p.q.-Baer rings) under which the formal power series ring $R[[x]]$ over $R$ is a right PP-ring (or left p.q.-Baer ring, respectively). For left APP-rings we have the following results.

Proposition 3.14. Let $R$ be a ring satisfying descending chain condition on left and right annihilators. If $R$ is a left APP-ring, then so is $R[[x]]$.

Proof. Suppose that $f(x)=a_{0}+a_{1} x+a_{2} x^{2}+\cdots$ and $g(x)=b_{0}+b_{1} x+$ $b_{2} x^{2}+\cdots \in R[[x]]$ are such that $f(x) \in l_{R[[x]]}(R[[x]] g(x))$. Then $f(x) R[[x]] g(x)=0$. Thus $f(x) \operatorname{Rg}(x)=0$. It follows that

$$
\sum_{i+j=k} a_{i} r b_{j}=0, \quad k=0,1,2, \ldots,
$$

where $r$ is an arbitrary element of $R$. Thus, since $a_{0} r b_{0}=0$, one has $a_{0} \in l_{R}\left(R b_{0}\right)$. So there exists $w_{0} \in l_{R}\left(R b_{0}\right)$ such that $a_{0}=a_{0} w_{0}$. Let $r^{\prime} \in R$ and take $r=w_{0} r^{\prime}$ in $a_{1} r b_{0}+a_{0} r b_{1}=0$. Then $a_{1} w_{0} r^{\prime} b_{0}+a_{0} w_{0} r^{\prime} b_{1}=0$. But $a_{1} w_{0} r^{\prime} b_{0}=0$. So $a_{0} w_{0} r^{\prime} b_{1}=0$. Since $a_{0}=a_{0} w_{0}$, we have $a_{0} r^{\prime} b_{1}=0$, which implies that $a_{0} \in l_{R}\left(R b_{1}\right)$. Also $a_{1} r b_{0}=0$ for any $r \in R$. This means that $a_{1} \in l_{R}\left(R b_{0}\right)$.

Now assume that

$$
a_{i} \in l_{R}\left(R b_{j}\right), \quad i+j=0,1,2, \ldots, k-1 .
$$


Then, since $R$ is a left APP-ring, there exists $w_{0} \in l_{R}\left(R b_{0}\right)$ such that $a_{i}=a_{i} w_{0}, i=$ $0,1, \ldots, k-1$. Let $r^{\prime} \in R$ and take $r=w_{0} r^{\prime}$ in $\sum_{i+j=k} a_{i} r b_{j}=0$. Then, since $a_{k} w_{0} r^{\prime} b_{0}=$ 0 , we have

$$
a_{0} w_{0} r^{\prime} b_{k}+a_{1} w_{0} r^{\prime} b_{k-1}+\cdots+a_{k-1} w_{0} r^{\prime} b_{1}=a_{0} r^{\prime} b_{k}+a_{1} r^{\prime} b_{k-1}+\cdots+a_{k-1} r^{\prime} b_{1}=0 .
$$

From $a_{0}, a_{1}, \ldots, a_{k-2} \in l_{R}\left(R b_{1}\right)$ it follows that there exists $w_{1} \in l_{R}\left(R b_{1}\right)$ such that $a_{i}=$ $a_{i} w_{1}, i=0,1, \ldots, k-2$. Let $y \in R$ and take $r^{\prime}=w_{1} y$. Then, since $a_{k-1} w_{1} y b_{1}=0$, we have

$$
a_{0} w_{1} y b_{k}+a_{1} w_{1} y b_{k-1}+\cdots+a_{k-2} w_{1} y b_{2}=a_{0} y b_{k}+a_{1} y b_{k-1}+\cdots+a_{k-2} y b_{2}=0 .
$$

Continuing in this manner, we have $a_{0} c b_{k}=0$, where $c$ is an arbitrary element of $R$. This implies that $a_{1} c b_{k-1}=0, \ldots, a_{k-1} c b_{1}=0, a_{k} c b_{0}=0$. Thus

$$
a_{0} \in l_{R}\left(R b_{k}\right), \quad a_{1} \in l_{R}\left(R b_{k-1}\right), \ldots, a_{k} \in l_{R}\left(R b_{0}\right) .
$$

Therefore, by the induction principle, we have shown that $a_{i} \in l_{R}\left(R b_{j}\right), i, j=$ $0,1, \ldots$.

Consider the descending chain as following:

$$
l_{R}\left(R b_{0}\right) \supseteq l_{R}\left(R b_{0}+R b_{1}\right) \supseteq l_{R}\left(R b_{0}+R b_{1}+R b_{2}\right) \supseteq \cdots .
$$

Then there exists $m$ such that $l_{R}\left(R b_{0}+R b_{1}+\cdots+R b_{m}\right)=l_{R}\left(R b_{0}+R b_{1}+\cdots+\right.$ $\left.R b_{m}+R b_{m+1}\right)=\cdots$. On the other hand, by considering the descending chain as following:

$$
r_{R}\left(a_{0}\right) \supseteq r_{R}\left(a_{0}, a_{1}\right) \supseteq r_{R}\left(a_{0}, a_{1}, a_{2}\right) \supseteq \cdots,
$$

there exists $n$ such that $r_{R}\left(a_{0}, a_{1}, \ldots, a_{n}\right)=r_{R}\left(a_{0}, a_{1}, \ldots, a_{n}, a_{n+1}\right)=\cdots$. Since $a_{0}$, $a_{1}, \ldots, a_{n} \in l_{R}\left(R b_{0}+R b_{1}+\cdots+R b_{m}\right)$, by Proposition 2.6, there exists $c \in l_{R}\left(R b_{0}+\right.$ $\left.R b_{1}+\cdots+R b_{m}\right)$ such that $a_{i}=a_{i} c$ for $i=0,1, \ldots, n$. Thus $1-c \in r_{R}\left(a_{0}, a_{1}, \ldots, a_{n}\right)$. So $1-c \in r_{R}\left(a_{0}, a_{1}, \ldots, a_{n}, \ldots, a_{k}\right)$ for any $k \geq n$, which implies that $a_{k}=a_{k} c$ for any $k \geq n$. Now it is easy to see that $f(x)=f(x) c$ and $c \in l_{R[[x]]}(R[[x]] g(x))$. This shows that $R[[x]]$ is a left APP-ring.

A ring $R$ is said to be $\aleph_{0}$-self-injective if each $R$-homomorphism from a countably generated left ideal $L$ of $R$ into $R$ is induced by multiplication by an element of $R$. $R$ is said to be left duo if every left ideal of $R$ is two-sided.

PROPOSITION 3.15. Let $R$ be a reduced left duo ring which is $\aleph_{0}$-self-injective. If $R$ is a left APP-ring, then so is $R[[x]]$.

Proof. Suppose that $f(x)=a_{0}+a_{1} x+a_{2} x^{2}+\cdots$ and $g(x)=b_{0}+b_{1} x+$ $b_{2} x^{2}+\cdots \in R[[x]]$ are such that $f(x) \in l_{R[[x]]}(R[[x]] g(x))$. Then $f(x) R[[x]] g(x)=0$. Thus $f(x) \operatorname{Rg}(x)=0$. By analogy with the proof of Proposition 3.14, we have $a_{i} R b_{j}=0$ for all $i$ and $j$. Let $I=\sum_{i=0}^{\infty} R a_{i}, J=\sum_{i=0}^{\infty} R b_{i}$. Then $I J=0$. Hence $I \bigcap J=0$ since $R$ is reduced. Therefore, the projection map $\alpha: I \oplus J \longrightarrow R$ via $\alpha(a+b)=a, a \in I, b \in J$, is well-defined and by hypothesis, $\alpha$ is given by multiplication by an element $c \in R$. Now for every $a \in I, a=\alpha(a+b)=(a+b) c=a c+b c$. Since $R$ is a left duo ring, we have $a=a c$ for every $a \in I$ and $b c=0$ for every $b \in J$. Since $R$ is reduced, it follows that $c R b_{i}=0, i=0,1,2, \ldots$ Thus $f(x)=f(x) c$ and $c \in l_{R[x]]}(R[[x]] g(x))$. This shows that $R[[x]]$ is a left APP-ring. 
ACKNOWLEDGment. The authors wish to express their sincere thanks to the referee for valuable suggestions and especially for improvement of Proposition 2.9 and Corollary 2.10 .

\section{REFERENCES}

1. D. D. Anderson and V. Camillo, Armendariz rings and Gaussian rings, Comm. Algebra 26 (1998), 2265-2272.

2. E. P. Armendariz, A note on extensions of Baer and p.p.-rings, J. Austral. Math. Soc. 18 (1974), 470-473.

3. G. F. Birkenmeier, J. Y. Kim and J. K. Park, A sheaf representation of quasi-Baer rings, J. Pure Appl. Algebra 146 (2000), 209-223.

4. G. F. Birkenmeier, J. Y. Kim and J. K. Park, On quasi-Baer rings, Contemp. Math. 259 (2000), 67-92.

5. G. F., Birkenmeier, J. Y. Kim and J. K. Park, On polynomial extensions of principally quasi-Baer rings, Kyungpook Math. J. 40 (2000), 247-254.

6. G. F. Birkenmeier, J. Y. Kim and J. K. Park, Principally quasi-Baer rings, Comm. Algebra 29 (2001), 639-660.

7. G. F. Birkenmeier and J. K. Park, Triangular matrix representations of ring extensions, J. Algebra 265 (2003), 457-477.

8. J. W. Brewer, Power series over commutative rings (Marcel Dekker, 1981).

9. W. E. Clark, Twisted matrix units semigroup algebras, Duke Math. J. 34 (1967), 417-423.

10. J. A. Fraser and W. K. Nicholson, Reduced PP-rings, Math. Japonica 34 (1989), 715-725.

11. Y. Hirano, On annihilator ideals of a polynomial ring over a noncommutative ring, J. Pure Appl. Algebra 168 (2002), 45-52.

12. C. Huh, H. K. Kim and Y. Lee, p.p. rings and generalized p.p. rings, J. Pure Appl. Algebra 167 (2002), 37-52.

13. I. Kaplansky, Rings of Operators (W. A. Benjamin, 1968).

14. Z. K. Liu and J. Ahsan, PP-rings of generalized power series, Acta Math. Sinica, English Series 16 (2000), 573-578.

15. Z. K. Liu, A note on principally quasi-Baer rings, Comm. Algebra 30 (2002), 3885-3890.

16. J. Okninski, Semigroup algebras (Marcel Dekker, 1991).

17. L. Oyonarte and B. Torrecillas, ふ-products of injective objects in Grothendieck categories, Comm. Algebra 25 (1997), 923-934.

18. D. S. Passman, The algebraic structure of group rings (Wiley, 1977).

19. B. Stenstrom, Rings of quotients (Springer-Verlag, 1975).

20. M. L. Teply, Large subdirect products, Proc. Inter. Conf. Ring Theory, Granada, Spain, Lecture Notes in Mathematics 1328 (Springer, 1986), 283-304.

21. M. L. Teply, Semicocritical modules (University of Murcia Press, 1987).

22. H. Tominaga, On s-unital rings, Math. J. Okayama Univ. 18 (1976), 117-134.

23. A. Tuganbaev, Rings close to regular (Kluwer, 2002). 